\title{
The contribution of lifestyle coaching of overweight patients in primary care to more autonomous motivation for physical activity and healthy dietary behaviour: results of a longitudinal study
}

Geert M Rutten ${ }^{1 *}$, Jessie JM Meis ${ }^{1}$, Marike RC Hendriks², Femke JM Hamers ${ }^{3}$, Cindy Veenhof ${ }^{4}$ and Stef PJ Kremers ${ }^{1}$

\begin{abstract}
Background: Combined lifestyle interventions (CLIs) have been advocated as an effective instrument in efforts to reduce overweight and obesity. The odds of maintaining higher levels of physical activity (PA) and healthier dietary behaviour improve when people are more intrinsically motivated to change their behaviour. To promote the shift towards more autonomous types of motivation, facilitator led CLIs have been developed including lifestyle coaching as key element. The present study examined the shift in types of motivation to increase PA and healthy dieting among participants of a primary care CLI, and the contribution of lifestyle coaching to potential changes in motivational quality.

Methods: This prospective cohort study included participants of 29 general practices in the Netherlands that implemented a CLI named 'BeweegKuur'. Questionnaires including items on demographics, lifestyle coaching and motivation were sent at baseline and after 4 months. Aspects of motivation were assessed with the Behavioural Regulation and Exercise Questionnaire (BREQ-2) and the Regulation of Eating Behaviour Questionnaire (REBS). We performed a drop out analysis to identify selective drop-out. Changes in motivation were analysed with t-tests and effect size interpretations (Cohen's $d$ ), and multivariate regression analysis was used to identify predictors of motivational change.
\end{abstract}

Results: For physical activity, changes in motivational regulation were fully in line with the tenets of Self Determination Theory and Motivational Interviewing: participants made a shift towards a more autonomous type of motivation (i.e. controlled types of motivation decreased and autonomous types increased). Moreover, an autonomy supportive coaching style was generally found to predict a larger shift in autonomous types of motivation. For healthy dietary behaviour, however, except for a small decrease in external motivation, no favourable changes in different types of motivation were observed. The relation between coaching and motivation appeared to be influenced by the presence of physical activity guidance in the programme.

Conclusions: Motivation of participants of a real life primary care CLI had changed towards a more autonomous motivation after 4 months of intervention. Autonomy-supportive lifestyle coaching contributed to this change with respect to physical activity. Lifestyle coaching for healthy diet requires thorough knowledge about the problem of unhealthy dieting and solid coaching skills.

\footnotetext{
*Correspondence: g.rutten@maastrichtuniversity.nl

${ }^{1}$ School for Nutrition, Toxicology and Metabolism (NUTRIM), Department of

Health Promotion, Maastricht University, Maastricht, The Netherlands

Full list of author information is available at the end of the article
} 


\section{Background}

Combined lifestyle interventions (CLIs) in primary care, including dietary advice and physical activity, have been advocated as an effective instrument in efforts to reduce the growing problem of overweight and obesity [1-3]. Enhanced levels of physical activity and a healthier diet maintained over a longer period of time have shown to be associated with better health outcomes for obese individuals [3-5]. However, low enrolment rates, high dropout rates and incomplete implementation have limited the effectiveness of CLIs in real life situations [6,7]. In contrast to reaching immediate, short-term changes [8-10], it has proved difficult to achieve sustained behaviour change, which is required to prevent weight regain and chronic diseases such as type 2 diabetes or cardiovascular diseases among obese individuals $[11,12]$.

Research has demonstrated that the probability of maintaining higher levels of physical activity and healthier dietary behaviour improve when people are more intrinsically motivated to change their behaviour [13-15]. In a recent review on motivation and self-regulation in relation to weight reduction [15], the authors indicated that interventions may so far have focused too much on influencing cognitions and skills and ignored the importance of perceived autonomy in the process of adopting new behaviours [15].

Intrinsic motivation is the most pronounced type of autonomous motivation described in Self-Determination Theory (SDT)[16,17]. This theory distinguishes 3 types of motivation: amotivation, extrinsic motivation and intrinsic motivation. Extrinsic motivation is subdivided into four types of motivational regulation, viz. two controlled types, external and introjected regulation and two autonomous types, identified and integrated regulation. The theory indicates that the quality of the motivation to engage in a certain behaviour can shift from amotivation and/or more controlled types of motivational regulation towards the autonomous types of regulation and towards the ultimate form of autonomous motivation, intrinsic motivation. To reach this shift, SDT indicates that there are three basic needs, i.e. autonomy, competence and relatedness, that should be supported. If individuals experience an insufficient level of one of these needs it hampers the shift towards autonomous motivation.

To promote the shift in motivation towards the more autonomous types, facilitator-led CLIs have been developed. An example is the Dutch BeweegKuur intervention, which, in addition to physical activity support and dietary advice, includes lifestyle coaching by means of motivational interviewing $[18,19]$. Through the combination of these three components the intervention touches on the need for autonomy by means of lifestyle coaching, on competence by means of lifestyle coaching and physical activity and dietary behaviour guidance and on relatedness by means of group sessions. By the inclusion of autonomy supportive lifestyle coaching (LSC), the intervention intends to produce sustainable changes in energy balance related behaviours. The objective of the intervention is to enhance overweight or obese participants' levels of physical activity and improve their dietary behaviour. The BeweegKuur intervention distinguishes 3 programmes that all include 7 lifestyle coaching sessions and 2 individual and 5 group sessions with a dietician. The programmes differ in the extent and intensity of physical activity support. The Independent exercise programme includes no physical activity support by a physical therapist (PT), while the Startup programme includes six PT sessions in 3-4 months and the Supervised exercise programme includes 3-4 months of intensive PT-guided training at least twice a week. Individuals are assigned to the programmes on the basis of their weight-related health risk, which is based on their BMI, their waist circumference and the presence of risk factors for type 2 Diabetes or Cardio Vascular Disease, or of comorbidities. A low or moderate level of physical activity is also an inclusion criterion for the BeweegKuur intervention. The LSC carries out the primary assessment and includes people in the intervention. LSCs involved in the BeweegKuur intervention, which in most cases are general practitioner assistants and sometimes physical therapists, are trained in motivational interviewing (MI), a method for autonomy-supportive coaching [20,21].

In their review, Teixeira et al. [15] demonstrate that, despite the importance of autonomous motivation for sustained behaviour change and despite the fact that it is the primary focus of its application, few studies on motivational interviewing and weight loss have considered changes in the quality of motivation as an outcome.

The present study aimed to assess the shift in quality of motivation to increase physical activity and to engage in a healthier diet among participants of the BeweegKuur intervention. In addition the study aimed to examine the contribution of lifestyle coaching to potential changes in motivational quality. Given the autonomy supportive character of MI, it was expected that the MI aspects of the LSC's counseling style would reduce controlled types of motivation and increase autonomous types of motivation.

\section{Methods}

\section{Design and recruitment}

In this prospective multicentre cohort study, data were gathered from a sample of 29 out of 150 BeweegKuur locations. The 29 locations were spread geographically across the Netherlands. Data were collected using a longitudinal questionnaire survey with two measurements: at baseline and at 4-months follow-up.

All locations included a general practitioner (GP), a GP assistant, a physical therapist and a dietician. The 
GP preselected potential participants and referred them to the LSC. The LSC had one to three sessions with the participant before the latter made a decision on whether to enroll in the BeweegKuur intervention. LSCs were allowed to include a maximum of 20 participants for every programme. After the participant had given informed consent, the LSC handed them the baseline questionnaire. Further questionnaires were sent to participants directly by the researchers by mail, while a web based version was available for those who preferred it. To reduce the rate of non-response, participants were reminded after two weeks by email and after 4 weeks by phone, or by another email if they were not reachable by phone. The study was approved by the Medical Review Ethics Committee (MEC) azM-UM (File no. NL 32615.068.10/MEC 10-3-051).

\section{Measurements}

\section{Background variables}

The questionnaires assessed demographic variables (age and gender), educational level (high, medium, low) and employment status (paid job or not). Self-reported BMI was established by asking participants to fill in their body length and weight.

\section{Motivational regulation}

The quality of motivation for physical activity was assessed using the Behavioural Regulation and Exercise Questionnaire (BREQ-2) [22], including four complementary items on integrated regulation [23]. Answers were given on a 5 -point Likert scale (strongly disagree to strongly agree). Cronbach's alpha reliability coefficients for the BREQ-2 subscales in the present study varied from 0.73 to 0.91 . In accordance with a previous study [24], the item 'Because I get restless if I don't exercise regularly', was removed from the identified regulation subscale, since reliability analysis indicated that it detracted from the internal consistency of the subscale.

Quality of motivation for healthy nutrition was measured with an abbreviated 12-item Dutch version of the Regulation of Eating Behaviours Scale (REBS) [25]. All motivational regulations were measured by 2 items with a 5-point Likert answering scale (strongly disagree to strongly agree). Cronbach's alpha reliability coefficients for the REBS subscales in the present study varied from 0.61 to 0.84

\section{Lifestyle coaching}

Participants' experiences with the LSC were assessed by means of questions developed using the BeweegKuur LSC protocol as a lead [26]. This protocol describes the actions the LSC is expected to perform and includes a manual for motivational interviewing. Questions were developed by the primary research group and reviewed by members of the advisory board, after which the questions were adjusted in accordance with the comments. This resulted in 21 questions concerning the LSC, 10 of which concerned concrete actions, e.g. 'performed body measurements' or 'referred to physical therapist', and 11 concerned the LSC's communication style, e.g. 'made me realize that my diet is unhealthy' or 'took the decision to participate in the BeweegKuur for me'. Answers were scored on a 5 point Likert scale (completely disagree to completely agree). A principal component analysis (oblimin rotation, 25 iterations) revealed that participants had experienced 3 main categories in coaching styles (See Additional file 1). Three types of lifestyle coaching were distinguished: autonomy-supportive, in which the LSC helps and supports the participants in making their own decisions (7 items, Cronbach's $\alpha=0.74)$, controlling, in which the LSC makes the decisions for the participants ( 2 items, Cronbach's $\alpha=0.66$ ), and protocol adherent, which means that the LSC applies the tests recommended in the protocol (6 items, Cronbach's $\alpha=0.65$ ).

\section{Drop-out analysis}

Binary logistic regression analysis with drop-out as the dependent variable (non drop-outs $=0$, drop-outs $=1$ ) was used to identify selective drop-out. The demographic variables gender, age and educational level were included as covariates, together with the programme variables and the types of motivation for physical activity and healthy diet.

\section{Data analysis}

Prior to the analyses, the database was checked for outliers and missing data and 'cleaned'. In case of anomalous data (for instance multiple boxes checked were only one was requested), the questionnaires were checked again, and if that did not lead to greater clarity, the item was scored as missing. Parametric tests were only performed if data had a normal distribution. In the various analyses missing data were handled as system missing. In general, analyses included 255 to 290 (>85\%) of 298 participants due to missing data, showing that the amount of missing data was limited.

We used descriptive analysis for the demographic variables. Differences in scores for the various types of motivational regulation between programmes at baseline were analysed with one-way ANOVA with post-hoc analysis. T-test for dependent samples was used to assess changes in motivation between baseline and 4 months follow-up. Changes were expressed in effect sizes (ES) using Cohen's d. In accordance with Cohen's classification [27] we categorized an ES of 0.2 as small, ES of 0.5 as medium and $\mathrm{ES} \geq 0.8$ as large.

Relations between types of lifestyle coaching and changes in motivational regulation were analysed with multiple linear regression analyses (backward method). 
The types of motivation (i.e. amotivation, external, introjected, identified and integrated regulation and intrinsic motivation) were subsequently included as the dependent variable in the analyses. The demographic variables gender, age and educational level, lifestyle coaching, and the motivation scores at baseline were included in the model. Since participants were assigned to one of the three BeweegKuur programmes that differed in their physical activity guidance, we also added the BeweegKuur programmes as dummy variables to the equation (with the Independent exercise programme as reference). After interpretation of the results of these analyses we explored the possibility of interaction between programme and autonomy supportive or controlled coaching. For that purpose we added four interaction terms (programme * coaching) to the equation. All analyses were performed with SPSS 20 for Windows with $\alpha=0.05$.

\section{Results}

\section{Characteristics of participants}

At baseline 409 participants were included, 72.9\% ( $\mathrm{n}=$ 298) of whom also completed the follow-up questionnaire. The average age of these 298 participants was 55.3 years old $(\mathrm{SD}=12.2)$ and $64.8 \%$ were female. The mean BMI was $32.9 \mathrm{~kg} / \mathrm{m}^{2}$ with $73.0 \%$ of the participants being obese. A low educational level was reported by $35.9 \%$, a high level by $22.4 \%$, and $47.3 \%$ had a paid job. ANOVA tests showed no significant baseline differences between participants of the three different programmes as regards types of motivation for physical activity $(0.208 \leq \mathrm{p} \leq 0.945)$ or for healthy dietary behaviour $(0.091 \leq \mathrm{p} \leq 0.993)$.

\section{Drop-out}

The results of the drop-out analysis showed that neither the demographic variables, nor the programme that participants were assigned to, were predictors of drop-out. Baseline types of motivation for physical activity were not significantly related to drop-out either. As regards healthy diet, a higher level of integrated motivational regulation at baseline predicted a lower odds of drop-out $(\mathrm{OR}=0.66$, $\mathrm{p}=.037)$.

\section{Motivation for physical activity and healthy dietary behaviour}

Amotivation $\left(\mathrm{ES}={ }^{-} 0.23\right)$ and external regulation $\left(\mathrm{ES}={ }^{-} 0.16\right)$ for physical activity decreased significantly between baseline and 4 months (Table 1 ) with a small effect size. Integrated regulation and intrinsic motivation increased, with a small to moderate effect size $(E S=0.36$ and 0.33 , respectively; Figure 1). As regards healthy dietary behaviour, only external motivation decreased significantly, with a small effect size (ES $=-0.13$; Figure 1$)$. All other types of motivation did not significantly change (Table 1 ).

\section{Longitudinal relation between lifestyle coaching and motivation \\ Physical activity}

As regards physical activity, a higher age predicted higher identified and integrated motivation (Table 2). A higher educational level predicted lower amotivation and lower introjected motivation at 4 months.

Autonomy-supportive lifestyle coaching for physical activity predicted lower amotivation and higher external, identified and intrinsic motivation. Controlled lifestyle coaching predicted higher amotivation and lower identified

Table 1 Changes in motivation for physical activity and healthy diet between baseline and 4 months $n \geq 279$; T1 = 4 months

\begin{tabular}{|c|c|c|c|c|c|c|c|c|}
\hline Physical activity & $\begin{array}{l}\text { Mean } \\
\text { difference T1-T0 }\end{array}$ & SD & $95 \% \mathrm{Cl}$ & $\mathbf{t}$ & $\mathbf{P}$ & $\begin{array}{l}\text { Adj. mean } \\
\text { difference T1-T0 }\end{array}$ & SD & $\mathbf{P}$ \\
\hline intrinsic motivation & 0.328 & 0.908 & $0.223 ; 0.433$ & 6.131 & .000 & 0.350 & 0.130 & .000 \\
\hline integrated regulation & 0.344 & 0.899 & $0.239 ; 0.449$ & 6.425 & .000 & 0.355 & 0.160 & .000 \\
\hline identified regulation & 0.078 & 0.733 & $-0.007 ; 0.164$ & 1.823 & .071 & 0.073 & 0.083 & .000 \\
\hline introjected regulation & 0.051 & 1.180 & $-0.087 ; 0.189$ & 0.731 & .466 & 0.050 & 0.265 & .002 \\
\hline external regulation & -0.141 & 0.954 & $-0.252 ;-0.030$ & -2.507 & .013 & -0.131 & 0.202 & .000 \\
\hline amotivation & -0.188 & 0.960 & $-0.300 ;-0.075$ & -3.287 & .001 & -0.164 & 0.185 & .000 \\
\hline \multicolumn{9}{|l|}{ Healthy diet } \\
\hline intrinsic motivation & -0.084 & 0.811 & $-0.178 ; 0.010$ & -1.750 & .081 & -0.086 & 0.146 & .000 \\
\hline integrated regulation & 0.009 & 0.845 & $-0.090 ; 0.107$ & 0.175 & .861 & 0.002 & 0.157 & .860 \\
\hline identified regulation & -0.049 & 0.745 & $-0.135 ;-0.038$ & -1.109 & .268 & -0.035 & 0.105 & .000 \\
\hline introjected regulation & -0.002 & 1,108 & $-0.131 ; 0.127$ & -0.027 & .979 & 0.018 & 0.068 & .000 \\
\hline external regulation & -0.147 & 1.087 & $-0.273 ;-0.020$ & -2.284 & .023 & -0.131 & 0.160 & .000 \\
\hline amotivation & 0.011 & 0.889 & $-0.093 ; 0.114$ & 0.200 & .841 & 0.017 & 0.177 & .111 \\
\hline
\end{tabular}




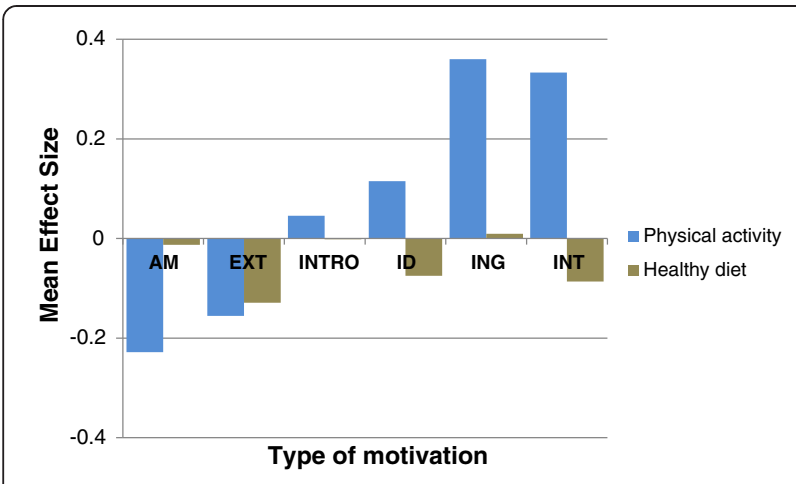

Figure 1 Changes in types of motivation for physical activity and healthy diet.

and integrated regulation. Higher levels of protocoladherent care by the LSC did not predict any change in the type of motivation.

Being included in the Startup exercise programme predicted lower amotivation and higher identified motivation at 4 months, whereas the Supervised exercise programme predicted higher identified and higher intrinsic motivation.

\section{Interaction between programme and type of coaching for} physical activity

We observed significant interaction effects on integrated motivation between the Start-up programme $(\mathrm{p}=0.031)$ as well as the Supervised exercise programme $(\mathrm{p}=0.007)$ and controlled coaching. An interaction effect was also observed for the Start-up programme with controlled coaching on identified motivation $(p=0.049)$. For all other interaction terms, no significant effects were found. Our subsequent analyses with stratification for programme revealed that higher levels of perceived controlled coaching predicted a lower level of integrated motivation for the Independent exercise programme $(p=0.001)$ and a lower level of identified motivation for the Independent $(\mathrm{p}=0.041)$ and Supervised exercise programme $(\mathrm{p}=0.010)$.

\section{Healthy diet}

As regards healthy diet, we found no relations between lifestyle coaching and any type of motivation for healthy diet. A higher educational level and participating in the Start up or Supervised exercise programme predicted lower amotivation (Table 3). Participating in these exercise programmes also predicted higher integrated motivation.

Table 2 Longitudinal relation between lifestyle coaching and motivation for physical activity

\begin{tabular}{|c|c|c|c|c|c|c|c|c|c|c|c|c|c|c|c|c|c|c|}
\hline \multirow[b]{2}{*}{$\begin{array}{l}\text { Independent } \\
\text { variables }\end{array}$} & \multicolumn{3}{|c|}{ Amotivation } & \multicolumn{3}{|c|}{$\begin{array}{l}\text { External } \\
\text { regulation }\end{array}$} & \multicolumn{3}{|c|}{$\begin{array}{l}\text { Introjected } \\
\text { regulation }\end{array}$} & \multicolumn{3}{|c|}{$\begin{array}{l}\text { Identified } \\
\text { regulation }\end{array}$} & \multicolumn{3}{|c|}{$\begin{array}{l}\text { Integrated } \\
\text { regulation }\end{array}$} & \multicolumn{3}{|c|}{$\begin{array}{l}\text { Intrinsic } \\
\text { motivation }\end{array}$} \\
\hline & B & $\beta$ & P & B & $\beta$ & $P$ & B & $\beta$ & $P$ & B & $\beta$ & $P$ & B & $\beta$ & $P$ & B & $\beta$ & P \\
\hline Constant & 1.267 & & .000 & -0.375 & & .273 & 1.425 & & .000 & 1.105 & & .001 & 1.077 & & .000 & 0.475 & & .193 \\
\hline
\end{tabular}

Demographic variables

Gender

(0 male,

1 female)

Age (Years)

Educational level $\quad-0.146 \quad-0.164 \quad .006$

$\begin{array}{llllll}0.008 & 0.148 & .008 & 0.010 & 0.127 & .012\end{array}$

(Low, Med, High)

Lifestyle coaching

\begin{tabular}{|c|c|c|c|c|c|c|c|c|c|c|c|c|c|c|c|c|c|c|}
\hline $\begin{array}{l}\text { Autonomy } \\
\text { supportive }\end{array}$ & -0.178 & -0.153 & .009 & 0.163 & 0.115 & .039 & & & & 0.139 & 0.121 & .030 & & & & 0.206 & 0.128 & .010 \\
\hline Controlled & 0.122 & 0.174 & .004 & & & & & & & -0.122 & -0.177 & .001 & -0.105 & -0.109 & .030 & & & \\
\hline \multicolumn{19}{|l|}{$\begin{array}{l}\text { Protocol } \\
\text { adherence }\end{array}$} \\
\hline \multicolumn{19}{|l|}{ Programmes } \\
\hline $\begin{array}{l}\text { Startup } \\
\text { programme }\end{array}$ & -0.328 & -0.204 & .005 & & & & & & & 0.249 & 0.158 & .020 & & & & & & \\
\hline $\begin{array}{l}\text { Supervised } \\
\text { programme }\end{array}$ & & & & & & & & & & 0.232 & 0.175 & .010 & & & & 0.230 & 0.124 & .043 \\
\hline \multicolumn{19}{|c|}{ Baseline motivation } \\
\hline $\begin{array}{l}\text { Corresponding } \\
\text { motivation at } \\
\text { baseline }\end{array}$ & 0.240 & 0.302 & .000 & 0.402 & 0.453 & .000 & 0.466 & 0.451 & .000 & 0.380 & 0.403 & .000 & 0.543 & 0.588 & .000 & 0.532 & 0.590 & .000 \\
\hline
\end{tabular}


Table 3 Longitudinal relation between lifestyle coaching and motivation for healthy diet

\begin{tabular}{|c|c|c|c|c|c|c|c|c|c|c|c|c|c|c|c|c|c|c|}
\hline \multirow[b]{2}{*}{ Independent variables } & \multicolumn{3}{|c|}{ Amotivation } & \multicolumn{3}{|c|}{$\begin{array}{l}\text { External } \\
\text { regulation }\end{array}$} & \multicolumn{3}{|c|}{$\begin{array}{l}\text { Introjected } \\
\text { regulation }\end{array}$} & \multicolumn{3}{|c|}{$\begin{array}{l}\text { Identified } \\
\text { regulation }\end{array}$} & \multicolumn{3}{|c|}{$\begin{array}{l}\text { Integrated } \\
\text { regulation }\end{array}$} & \multicolumn{3}{|c|}{$\begin{array}{l}\text { Intrinsic } \\
\text { motivation }\end{array}$} \\
\hline & B & $\beta$ & $P$ & B & $\beta$ & P & B & $\beta$ & $P$ & B & $\beta$ & $P$ & B & $\beta$ & $P$ & B & $\beta$ & $P$ \\
\hline Constant & 1.466 & & .000 & 1.116 & & .000 & 1.089 & & .000 & 1.546 & & .000 & 1.160 & & .000 & 0.802 & & .000 \\
\hline \multicolumn{19}{|l|}{ Demographic variables } \\
\hline \multicolumn{19}{|l|}{ Gender } \\
\hline \multicolumn{19}{|l|}{ (0 male, 1 female) } \\
\hline \multicolumn{19}{|l|}{ Age (Years) } \\
\hline $\begin{array}{l}\text { Educational level } \\
\text { (Low, Med, High) }\end{array}$ & -0.244 & -0.237 & .000 & & & & & & & & & & & & & & & \\
\hline
\end{tabular}

Lifestyle coaching

Autonomy supportive

Controlled

Protocol adherence

Programmes

Startup programme $\quad-0.364-0.197 \quad .005$

Supervised programme $\quad-0.292 \quad-0.188 \quad .007$

Baseline motivation

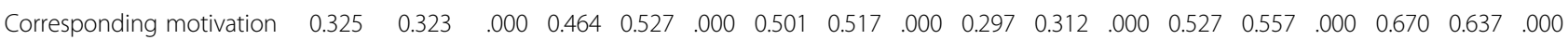
at baseline

$n=255$.

Interaction between programme and type of coaching for healthy diet

There was an interaction effect of the Start-up programme $(\mathrm{p}=0.002$ to 0.046$)$ as well as the Supervised exercise programme ( $p=0.004$ to 0.021$)$ and controlled coaching for all three types of autonomous motivation for healthy eating. We also observed an interaction effect of the Start-up programme and controlled coaching for introjected $(\mathrm{p}=0.019)$ and external motivation $(\mathrm{p}=0.008)$ and of the Supervised exercise programme and controlled coaching $(\mathrm{p}=0.006)$ on amotivation. In the stratified analyses higher perceived controlled coaching predicted a lower level of intrinsic $(p=0.014)$, integrated $(p \leq 0.001)$ and identified motivation $(p=0.002)$ for healthy eating of participants assigned to the Independent exercise programme. Higher levels of controlled coaching also predicted a higher external motivation for participants in the Start-up programme, and a higher level of amotivation for the Independent exercise $(p=0.001)$ and Start-up programme $(\mathrm{p}=0.035)$.

\section{Discussion}

This study examined the longitudinal relation between lifestyle coaching and changes in the different types of motivation in generally obese participants of a CLI. For physical activity, changes in motivational regulation were fully in line with the tenets of SDT and MI: participants showed a shift towards a more autonomous type of motivation (i.e. controlled types of motivation decreased and autonomous types increased). Moreover, if participants perceived an autonomy supportive coaching style, this was generally found to predict a larger shift in autonomous types of motivation. As regards healthy dietary behaviour, however, except for a small decrease in external motivation, no favourable changes in different types of motivation were observed. The level of perceived autonomy supportiveness of the Lifestyle Coaches appeared not to have induced any positive changes. An important predictor of favourable changes in autonomous motivation was the intensity of the BeweegKuur programmes.

Our finding of an improvement in autonomous types of motivation for physical activity confirms the findings of previous studies that investigated the relation between autonomous motivation and lifestyle changes in interventions for energy balance related behaviours [28,29]. The findings of our study specifically demonstrate that autonomy supportive lifestyle coaching in a 'real world' primary care CLI contributes to a favourable shift in motivational regulation for physical activity and that, in contrast, more controlled lifestyle coaching is related to higher amotivation and to a decrease of autonomous motivation.

However, in contrast to some previous studies [14,29], we did not observe this favourable pattern of changes in quality of motivation for healthy dietary behaviour. It has been argued before that changing dietary behaviour may involve some physical and psychological discomfort, making it hard to be intrinsically motivated to do it [30]. Participants in our study had a higher level of controlled 
types of motivation for healthy diet, compared to physical activity at baseline. Moreover, almost 75\% had losing weight as their main goal compared to $34 \%$ who chose health improvement as a goal. Previous studies revealed that higher controlled regulation of eating behaviours is related to poorer body image, lower psychological wellbeing [31], to a quantity focused eating regulation [32] and avoidance food planning [33]. Both strategies are negatively related to healthy eating behaviours [33]. Moreover it has been shown that physical appearance-focused (e.g. lose weight or gain a better physical appearance) instead of health-focused weight loss goals are related to less successful eating regulation strategies [30]. Furthermore, dietary behaviour may also include strong habits developed during childhood $[34,35]$. Such findings demonstrate the complexity of eating behaviour regulation and indicate that a more autonomous motivation is indeed required for successful eating behaviour regulation. However, as we observed in our study, it also indicates that obtaining this autonomous motivation may be rather difficult.

Our finding that the influence of coaching on motivation for physical activity was virtually absent when programmes included physical activity guidance, seems in line with findings of van Hoecke et al. (2014) [36]. Provision of a physical activity programme may facilitate the need for competence and may therefore be equally effective as need supportive coaching [36]. Our findings in the samples that were stratified by programme type also indicate that the negative influence of perceived controlled coaching on autonomous motivation for healthy eating may be neutralised when an intervention includes a physical activity component. Previous studies have suggested a clustering of personal determinants of diet and activity [37] and it has been demonstrated that autonomous exercise motivation may 'spill-over' to facilitate improvements in eating self-regulation [38].

Except for the intrinsic motivation of participants in the Independent exercise programme, we found no relation between autonomy supportive lifestyle coaching and improvement of autonomous motivation for healthy diet. The absence of this relation may be caused by the LSC's lack of knowledge about dietary behaviour and their insufficient skills to change it [39]. Previous failed attempts by participants to change their dietary behaviour may have resulted in a struggle and frustration with this behavioural goal, indicated by a lack of change in almost all types of the motivational pattern ("I want, I need and I must"). Failed attempts can cause frustration [40] and feelings of lack of competence, and would thus undermine one of the basic needs for autonomous motivation [41], if failures have repeatedly occurred. As a consequence the LSC should pay sufficient attention to improvement of feelings of competence.

The autonomy supportive coaching style we measured may not have fully covered the true breadth of autonomy support. Autonomy supportive coaching should include support of autonomy, competence and relatedness $[16,17]$. The items included in our autonomy supportive coaching style questionnaire however mainly concerned autonomy. It has been demonstrated before that primary care nurses find it difficult to apply autonomy-supportive coaching in lifestyle related behaviours [42-44] and with communication about nutritional behaviour in particular [45]. Although participants in our study indicated that they were very satisfied with the performance of the LSC, they gave substantially lower scores for the LSC's support to improve their dietary behaviour than the LSC's support to improve their physical activity. Given the previously mentioned complex nature of unhealthy dietary behaviour [46], the LCSs must feature thorough knowledge of the problem and highly developed MI skills to favourably influence the autonomous motivation to improve this behaviour.

Although external regulation decreased on a group level, we observed a positive relation between autonomysupportive coaching and an increase in external motivation for physical activity, which is not in accordance with SDT and MI. It has been observed before that people in treatment in general have a more external health locus of control [47] compared to those not in treatment. Moreover, our data revealed that participants judged the LSC to be very sympathetic and supportive. Participants with a higher external locus of control may have perceived the sympathy of the LSC as very rewarding, which may have induced an increase of their external motivation.

\section{Strengths and limitations}

Strengths of the current study include its theoretical foundation, longitudinal design, real-life intervention setting and use of validated questionnaires. The selfselected sample used in the study limits its external validity. We used a self-report questionnaire completed by participants as a proxy measure of LSC performance. This is a relatively cheap and manageable way to measure professional performance in primary care. Nevertheless, the validity of this measure would be served by direct observations, or in the ideal case by standardized patients, the gold standard in measurement of professional performance. However, both these alternatives are time-consuming and costly, especially in studies with larger numbers of participants $[48,49]$.

Although this study was neither designed nor executed as an effectiveness trial, we performed an intention to treat analysis with the last observation carried forward as well as with the group mean imputation method [50] to replace missing data in order to address potential bias in the study results due to loss to follow up. The results of both approaches however did not change the key findings of our study regarding the predictive value of 
perceived coaching style on participants' motivational regulation. However, it would require a randomized controlled trial to determine whether a change in coaching style would actually result in improved motivational regulation.

\section{Conclusion}

The results of this study show that the motivation of participants of a facilitator-led CLI had changed towards a more autonomous motivation after 4 months of intervention, and that autonomy-supportive lifestyle coaching contributed to this change with respect to physical activity. A physical activity component in a CLI seems to be important for autonomous motivation for physical activity as well as for healthy dietary behaviour. Lifestyle coaching for healthy diet requires thorough knowledge about the problem of unhealthy dieting and solid coaching skills. The results indicate that LSC's coaching skills were insufficient to shift the motivational regulation towards healthy diet in this generally obese population.

\section{Additional file}

Additional file 1: Items per type of coaching.

\section{Competing interests}

The authors declare that they have no competing interests.

\section{Authors' contributions}

GMR carried out the study and wrote the manuscript, JJMM cooperated in the execution of the study and in the data analysis. MRCH cooperated in the study and provided data and FJMH assisted in the execution of the study. $\mathrm{CV}$ was a member of the project advisory group and SPJK conceived the concept of the study and cooperated in the execution. All co-authors critically reviewed and contributed to the manuscript. All authors read and approved the final manuscript.

\section{Acknowledgement}

The study was supported by a grant from the Netherlands Organization for Health Research and Development (ZonMw): project number 123000003. The publication of the this paper was funded by the Netherlands Organization for Scientific Research (NWO).

\section{Author details}

${ }^{1}$ School for Nutrition, Toxicology and Metabolism (NUTRIM), Department of Health Promotion, Maastricht University, Maastricht, The Netherlands. ${ }^{2}$ School for Nutrition, Toxicology and Metabolism (NUTRIM), Department of Human Movement Science, Maastricht University, Maastricht, The Netherlands. ${ }^{3}$ Public Health Services (GGD) Southern Limburg, Geleen, The Netherlands. ${ }^{4}$ Netherlands Institute for Health Services Research (NIVEL), Utrecht, The Netherlands.

Received: 24 December 2013 Accepted: 27 June 2014 Published: 16 July 2014

\section{References}

1. Tuomilehto J, Lindstrom J, Eriksson JG, Valle TT, Hamalainen H, IlanneParikka P, Keinanen-Kiukaanniemi S, Laakso M, Louheranta A, Rastas M, Salminen V, Uusitupa M, Finnish Diabetes Prevention Study Group: Prevention of type 2 diabetes mellitus by changes in lifestyle among subjects with impaired glucose tolerance. N Engl J Med 2001, 344(18):1343-1350.

2. Knowler WC, Barrett-Connor E, Fowler SE, Hamman RF, Lachin JM, Walker EA, Nathan DM: Diabetes Prevention Program Research G: Reduction in the incidence of type 2 diabetes with lifestyle intervention or metformin. N Engl J Med 2002, 346(6):393-403.

3. Brown T, Avenell A, Edmunds LD, Moore H, Whittaker V, Avery L, Summerbell C: Systematic review of long-term lifestyle interventions to prevent weight gain and morbidity in adults. Obes Rev 2009, 10(6):627-638.

4. Gillett M, Royle P, Snaith A, Scotland G, Poobalan A, Imamura M, Black C, Boroujerdi M, Jick S, Wyness L, McNamee P, Brennan A, Waugh N: Nonpharmacological interventions to reduce the risk of diabetes in people with impaired glucose regulation: a systematic review and economic evaluation. Health Technol Assess 2012, 16(33):1-236. iii-iv.

5. Ho M, Garnett SP, Baur L, Burrows T, Stewart L, Neve M, Collins C: Effectiveness of lifestyle interventions in child obesity: systematic review with meta-analysis. Pediatrics 2012, 130(6):e1647-e1671.

6. Reinehr T, Widhalm K, L'Allemand D, Wiegand S, Wabitsch M, Holl RW: Two-year follow-up in 21,784 overweight children and adolescents with lifestyle intervention. Obesity (Silver Spring) 2009, 17(6):1196-1199.

7. Linmans JJ, Spigt MG, Deneer L, Lucas AE, de Bakker M, Gidding LG, Linssen $R$, Knottnerus JA: Effect of lifestyle intervention for people with diabetes or prediabetes in real-world primary care: propensity score analysis. BMC Fam Pract 2011, 12:95.

8. Wang X, Lyles MF, You T, Berry MJ, Rejeski WJ, Nicklas BJ: Weight regain is related to decreases in physical activity during weight loss. Med Sci Sports Exerc 2008, 40(10):1781-1788.

9. Goodpaster BH, Delany JP, Otto AD, Kuller L, Vockley J, South-Paul JE, Thomas SB, Brown J, McTique K, Hames KC, Lang W, Jakicic JM: Effects of diet and physical activity interventions on weight loss and cardiometabolic risk factors in severely obese adults: a randomized trial. J Am Med Assoc 2010, 304(16):1795-1802.

10. Unick JL, Beavers D, Jakicic JM, Kitabchi AE, Knowler WC, Wadden TA, Wing RR, Look ARG: Effectiveness of lifestyle interventions for individuals with severe obesity and type 2 diabetes: results from the Look AHEAD trial. Diabetes Care 2011, 34(10):2152-2157.

11. Wing RR, Venditti E, Jakicic JM, Polley BA, Lang W: Lifestyle intervention in overweight individuals with a family history of diabetes. Diabetes Care 1998, 21(3):350-359.

12. Wu T, Gao X, Chen M, Van Dam RM: Long-term effectiveness of diet-plusexercise interventions vs. diet-only interventions for weight loss: a meta-analysis. Obes Rev 2009, 10(3):313-323.

13. Silva MN, Markland D, Carraca EV, Vieira PN, Coutinho SR, Minderico CS, Matos MG, Sardinha LB, Teixeira PJ: Exercise Autonomous Motivation Predicts 3-yr Weight Loss in Women. Med Sci Sport Exer 2011, 43(4):728-737.

14. Shaikh AR, Vinokur AD, Yaroch AL, Williams GC, Resnicow K: Direct and mediated effects of two theoretically based interventions to increase consumption of fruits and vegetables in the Healthy Body Healthy Spirit trial. Health Educ Behav 2011, 38(5):492-501.

15. Teixeira PJ, Silva MN, Mata J, Palmeira AL, Markland D: Motivation, self-determination, and long-term weight control. Int J Behav Nutr Phy 2012, 9:22.

16. Deci EL, Ryan RM: A motivational approach to self: integration in personality. Nebr Symp Motiv 1990, 38:237-288.

17. Ryan RM, Deci EL: Self-determination theory and the facilitation of intrinsic motivation, social development, and well-being. Am Psychol 2000, 55(1):68-78.

18. Helmink JH, Meis JJ, de Weerdt I, Visser FN, de Vries NK, Kremers SP: Development and implementation of a lifestyle intervention to promote physical activity and healthy diet in the Dutch general practice setting: the BeweegKuur programme. Int J Behav Nutr Phys Act 2010, 7:49.

19. Berendsen BA, Hendriks MR, Verhagen EA, Schaper NC, Kremers SP, Savelberg HH: Effectiveness and cost-effectiveness of 'BeweegKuur', a combined lifestyle intervention in the Netherlands: rationale, design and methods of a randomized controlled trial. BMC Public Health 2011, 11:815.

20. Miller WR, Rollnick S: Motivational Interviewing. Helping people change, $3 d$ revised edn. New York: Guilford Publications; 2012.

21. Vansteenkiste M, Williams GC, Resnicow K: Toward systematic integration between self-determination theory and motivational interviewing as examples of top-down and bottom-up intervention development: autonomy or volition as a fundamental theoretical principle. Int I Behav Nutr Phys Act 2012, 9:23.

22. Ingledew DK, Wray JL, Markland D, Hardy L: Work-related goal perceptions and affective well-being. J Health Psychol 2005, 10(1):101-122. 
23. Wilson P, Rodgers W, Loitz C, Scime G: It's who I am... Really! The importance of integrated regulation in exercise context. J Appl Biobehav Res 2006, 11(2):79-104

24. Ingledew DK, Markland D: The role of motives in exercise participation. Psychol Health 2008, 23(7):807-828.

25. Pelletier LG, Dion SC, Slovinec-D'Angelo M, Reid R: Why do you regulate what you eat? Relationships between forms of regulation, eating behaviors, sustained dietary behavior change, and psychological adjustment. Motiv Emotion 2004, 28(3):245-277.

26. Handleiding BeweegKuur voor de Leefstijladviseur: Een gecombineerde leefstijlinterventie voor de (eerstelijns)zorg om mensen met een (zeer) hoog gewichtsgerelateerd gezondheidsrisico te begeleiden naar een gezonde leefstijl. Versie 1.2. http://www.beweegkuur.nl/documenten-2012/ handleidingen_protocol/handleiding-beweegkuur-leefstijladviseur.pdf.

27. Cohen J: Statistical power analysis for the behavioral sciences. Hillsdale, NJ: Lawrence Erlbaum Associates; 1988

28. Teixeira PJ, Silva MN, Coutinho SR, Palmeira AL, Mata J, Vieira PN, Carraca EV Santos TC, Sardinha LB: Mediators of weight loss and weight loss maintenance in middle-aged women. Obesity (Silver Spring) 2010, 18(4):725-735

29. Jacobs N, Hagger MS, Streukens S, De Bourdeaudhuij I, Claes N: Testing an integrated model of the theory of planned behaviour and selfdetermination theory for different energy balance-related behaviours and intervention intensities. Br J Health Psychol 2011, 16(Pt 1):113-134.

30. Verstuyf J, Patrick $H$, Vansteenkiste $M$, Teixeira PJ: Motivational dynamics of eating regulation: a self-determination theory perspective. Int J Behav Nutr Phys Act 2012, 9:21.

31. Carraca EV, Markland D, Silva MN, Coutinho SR, Vieira PN, Minderico CS, Sardinha LB, Teixeira PJ: Dysfunctional body investment versus body dissatisfaction: Relations with well-being and controlled motivations for obesity treatment. Motiv Emotion 2011, 35(4):423-434.

32. Pelletier LG, Dion SC: An examination of general and specific motivational mechanisms for the relations between body dissatisfaction and eating behaviors. J Soc Clin Psychol 2007, 26(3):303-333.

33. Otis N, Pelletier LG: Women's regulation styles for eating behaviors and outcomes: The mediating role of approach and avoidance food planning. Motiv Emotion 2008, 32:55-67.

34. Lally P, Chipperfield A, Wardle J: Healthy habits: efficacy of simple advice on weight control based on a habit-formation model. Int J Obes (Lond) 2008, 32(4):700-707.

35. McGowan L, Cooke L, Gardner B, Beeken RJ, Croker H, Wardle J: Healthy feeding habits: efficacy results from a cluster-randomized, controlled exploratory trial of a novel, habit-based intervention with parents. Am J Clin Nutr 2013, 98(3):769-777.

36. Van Hoecke AS, Delecluse C, Bogaerts A, Boen F: The long-term effectiveness of need-supportive physical activity counseling compared with a standard referral in sedentary older adults. J Aging Phys Act 2014, 22(2):186-198.

37. Kremers SPJ, De Bruijn GJ, Johannes Brug HS: Clustering of energy balance-related behaviours and their intrapersonal determinants. Psychol Health 2004, 19(5):595-606.

38. Mata J, Silva MN, Vieira PN, Carraca EV, Andrade AM, Coutinho SR, Sardinha $L B$, Teixeira PJ: Motivational "spill-over" during weight control: increased self-determination and exercise intrinsic motivation predict eating selfregulation. Health Psychol 2009, 28(6):709-716.

39. Wennberg AL, Lundqvist A, Hogberg U, Sandstrom H, Hamberg K: Women's experiences of dietary advice and dietary changes during pregnancy. Midwifery 2013, 29(9):1027-1034.

40. Weiner B: An attributional theory of achievement motivation and emotion. Psychol Rev 1985, 92(4):548-573.

41. Deci EL, Ryan RM: Intrinsic motivation and self-determination in human behavior. New York: Plenum; 1985.

42. Jansink R, Braspenning J, van der Weijden T, Elwyn G, Grol R: Primary care nurses struggle with lifestyle counseling in diabetes care: a qualitative analysis. BMC Fam Pract 2010, 11:41.

43. Noordman J, van Lee I, Nielen M, Vlek H, van Weijden T, van Dulmen S: Do trained practice nurses apply motivational interviewing techniques in primary care consultations? J Clin Med Res 2012, 4(6):393-401.

44. Noordman J, Koopmans B, Korevaar JC, van der Weijden T, van Dulmen S: Exploring lifestyle counselling in routine primary care consultations: the professionals' role. Fam Pract 2013, 30(3):332-340.
45. Van Dillen SM, Noordman J, Van Dulmen S, Hiddink GJ: Examining the content of weight, nutrition and physical activity advices provided by Dutch practice nurses in primary care: analysis of videotaped consultations. Eur J Clin Nutr 2014, 68(1):50-56.

46. Carter FA, Jansen A: Improving psychological treatment for obesity. Which eating behaviours should we target? Appetite 2012, 58(3):1063-1069.

47. Oliveira TH, Oliveira VC, Melo RC, Melo RM, Freitas AE, Ferreira PH: Patients in treatment for chronic low back pain have higher externalised beliefs: a cross-sectional study. Revista Brasileira Fisioterapia 2012, 16(1):35-39.

48. Luck J, Peabody JW: Using standardised patients to measure physicians' practice: validation study using audio recordings. BMJ 2002, 325:679-683.

49. Peabody JW, Luck J, Glassman P, Jain S, Hansen J, Spell M, Lee M: Measuring the quality of physician practice by using clinical vignettes: a prospective validation study. Ann Intern Med 2004, 141:771-780.

50. Israël A, Kuijvenhoven L, Van Der Laan J, Pannekoek J, Shulte Nordholt E: Imputatie 11. Den Haag: Centraal Bureau voor de Statistiek (CBS); 2011.

doi:10.1186/s12966-014-0086-z

Cite this article as: Rutten et al:: The contribution of lifestyle coaching of overweight patients in primary care to more autonomous motivation for physical activity and healthy dietary behaviour: results of a longitudinal study. International Journal of Behavioral Nutrition and Physical Activity 2014 11:86.

\section{Submit your next manuscript to BioMed Central and take full advantage of:}

- Convenient online submission

- Thorough peer review

- No space constraints or color figure charges

- Immediate publication on acceptance

- Inclusion in PubMed, CAS, Scopus and Google Scholar

- Research which is freely available for redistribution 